\title{
The associations between interleukin 10 polymorphisms and susceptibility to autoimmune uveitis - a meta-analysis
}

\author{
JAE HYUN JUNG, GWAN GYU SONG, JAE-HOON KIM, SUNG JAE CHOI
}

Division of Rheumatology, Korea University Medical Centre, Seoul, South Korea

\begin{abstract}
Autoimmune uveitis is an ocular inflammatory disease that is associated with genetic factors. Interleukin $10(I L-10)$ is an immune-regulatory cytokine of autoimmune diseases. IL-10 is considered a candidate gene for uveitis. We evaluate the association of IL-10 with susceptibility to autoimmune uveitis. The results from seven studies were pooled in the meta-analysis, covering a total of 2893 cases of uveitis and 4873 controls. Published literature from MEDLINE and Embase was retrieved. Meta-analyses were conducted on the associations between autoimmune uveitis and the $-1082 \mathrm{~A} / \mathrm{G}$ and -819 C/T polymorphisms of the IL-10 gene. The meta-analysis revealed no association between uveitis and the $I L-10-1082$ A allele $(O R=0.91,95 \% C I=0.64-1.30, p=0.62)$. The recessive, dominant, and homozygous models of the IL-10 -1082 A/G allele also suggested no association between autoimmune uveitis and each genotype. The meta-analysis revealed significant association between uveitis and the $-892 \mathrm{C}$ allele $(O R=0.81,95 \% C I=0.67-0.98, p=0.03)$. In addition, significant association was found in homozygous models $(O R=0.58,95 \% C I=0.36-0.92, p=0.02)$. However, the dominant and recessive models of the IL-10 -819 C/T polymorphisms showed no association between uveitis and each genotype. This meta-analysis showed that the $-1082 \mathrm{~A} / \mathrm{G}$ polymorphisms of IL-10 were not associated with autoimmune uveitis, but the -819 C/T polymorphisms were significantly associated with uveitis.
\end{abstract}

Key words: polymorphism, meta-analysis, interleukin-10, autoimmune uveitis.

(Centr Eur J Immunol 2019; 44 (3): 246-252)

\section{Introduction}

Uveitis is an inflammatory disease of the uvea, which includes three highly vascularised and pigmented areas: the iris, ciliary body, and choroid [1]. Uveitis is an important ocular disease because it is one of the leading causes of blindness next to cataract, glaucoma, diabetic retinopathy, and age-related macular degeneration [2]. It can result from both infectious and non-infectious or autoimmune causes. Autoimmune uveitis is often accompanied by a systemic inflammatory condition, such as Behçet's disease (BD), ankylosing spondylitis (AS), and Vogt-Koyanagi-Harada (VKH) disease [3].

Previous studies indicate that cytokines affect the development of autoimmune uveitis; biologic agents such as tumour necrosis factor $\alpha$ (TNF- $\alpha$ ) blocker have been used to treat uveitis [4-6]. Interleukin 10 (IL-10) is an immunosuppressive cytokine that inhibits chemokine production [7]. IL-10 promotes immune tolerance, resolution of inflammation, and apoptosis in both systemic and ocular diseases [8]. IL-10 polymorphisms are also associated with autoimmune diseases that can involve ocular symptoms such as AS and BD [9, 10]. IL-10 is considered a strong candidate gene for ocular inflammatory disease based on its chromosomal location and functional relevance. This cytokine contains three of the most well characterised single-nucleotide polymorphisms (SNPs): -1082 A/G (rs1800896), -819 C/T (rs1800871), and -592 C/A (rs1800872) [10].

Autoimmune uveitis has an unknown aetiology. Some uveitic entities are associated with systemic autoimmune diseases, whereas others are limited to the eye [11]. Uveitis is classified anatomically as anterior, intermediate, posterior, or panuveitis, and the location of the inflammation depends mainly on the type of systemic disease. Although the features of uveitis vary, they all develop due to ocular inflammation and inflammatory processes. IL-10, an anti-inflammatory cytokine, may affect the development of uveitis. Therefore, we investigated the genetic associations between IL-10 polymorphisms and the susceptibility to autoimmune uveitis, using a meta-analysis.

Correspondence: Prof. Sung Jae Choi, Division of Rheumatology, 73 Inchon-ro, Seong buk-gu, 02841 Seoul, South Korea, e-mail: csjmd888@korea.ac.kr

Submitted: 9.04.2017; Accepted: 29.05.2017 


\section{Material and methods}

\section{Study selection}

We searched MEDLINE and Embase for studies of the association between IL-10 and uveitis using the terms (interleukin-10 OR IL-10) AND (uveitis OR ocular inflammation OR autoimmune uveitis) AND (polymorphisms OR variant OR mutation OR genotype) in articles published through November 2016. References cited in the retrieved articles were also screened manually. No restrictions were placed on race, language, ethnicity, or geographic area.

\section{Inclusion and exclusion criteria}

The inclusion criteria for this investigation included case-control studies that determined the distributions of the IL-10 -1082 A/G, -819 C/T, and -592 C/A polymorphisms and uveitis, and detailed data for both the case and control groups, or any other data from which the desired numbers could be calculated. Studies were excluded based on the following criteria: those that contained overlapping data; studies in which the number of null and wild genotypes or alleles could not be ascertained; and investigations that were review articles or only contained abstracts.

\section{Data extraction}

The following information was obtained from each article: the first author, year of publication, ethnicity of the study population, demographics, numbers of cases and controls, Hardy-Weinberg equilibrium (HWE), and allele and genotype frequencies of the IL-10 -1082 A/G, $-819 \mathrm{C} / \mathrm{T}$, and -592 C/A alleles. Because IL-10 -819 C/T and -592 C/A exhibit complete linkage disequilibrium and data of $-819 \mathrm{C} / \mathrm{T}$ were larger in number than those of $-592 \mathrm{C} / \mathrm{A}$, we excluded the $-592 \mathrm{C} / \mathrm{A}$ in this meta-analysis. In cases of duplicated publications from the same study group, the study with a larger sample size was retained. This meta-analysis was reported based on the Preferred Reporting Items for Systemic Reviews and Meta-Analyses (PRISMA) guidelines [12].

\section{Statistical analyses}

The raw data for allele contrast and genotype frequencies without adjustment were used for the meta-analysis. A chi-squared test was conducted to detect whether the controls in each study conformed to HWE. The strength of each association between IL-10 polymorphism and susceptibility to autoimmune uveitis was estimated using the crude odds ratio (OR) and $95 \%$ confidence interval (CI).

The meta-analyses were performed on the following genetic models: allelic contrast, recessive (-1082 A/G: AA vs. AG + GG; $-892 \mathrm{C} / \mathrm{T}$ : CC vs. CT + TT), dominant (-1082 A/G: AA + AG vs. GG; $-892 \mathrm{C} / \mathrm{T}: \mathrm{CC}+\mathrm{CT}$ vs. TT), and homozygous (-1082 A/G: AA vs. GG; $-892 \mathrm{C} / \mathrm{T}$ : CC vs. TT).

Heterogeneity between studies was assessed with the Cochran $\mathrm{Q}$ test, in which a $p$-value $<0.10$ was consid- ered as statistically significant heterogeneity, and the heterogeneity among studies was tested by the $I_{c}$ statistic, in which $I_{c}>50 \%$ was considered as statistically significant heterogeneity [13]. A fixed-effect model was employed in cases with no significant heterogeneity [14]. Otherwise, a random-effect model was used [15]. Forest plots were drawn to visualise the overall effect. Data were analysed using Review Manager (RevMan) version 5.3 (The Nordic Cochrane Centre, The Cochrane Collaboration, 2014).

\section{Results \\ Study characteristics}

In total, 90 studies were identified through our electronic and manual searches. Of these investigations, 26 were selected for full-text review based on their titles and abstracts. Nineteen studies were excluded because they were review articles or meta-analyses, had the potential for overlapping data, lacked genotype data or suitable control data, or contained other polymorphisms. Thus, seven studies met the inclusion criteria (Fig. 1). Because two of these studies contained data on two different groups, we analysed these groups independently [16, 17]. Finally, this meta-analysis considered nine separate comparisons, including 2893 patients and 4873 controls [8, 16-21].

Two studies (three different groups) contained only minor allele data $[8,17]$. Thus, in the meta-analysis for -1082 $\mathrm{A} / \mathrm{G}$, allelic contrast and recessive and homozygous models included five studies, and the dominant model included seven studies. In the meta-analysis for $-819 \mathrm{C} / \mathrm{T}$, allelic

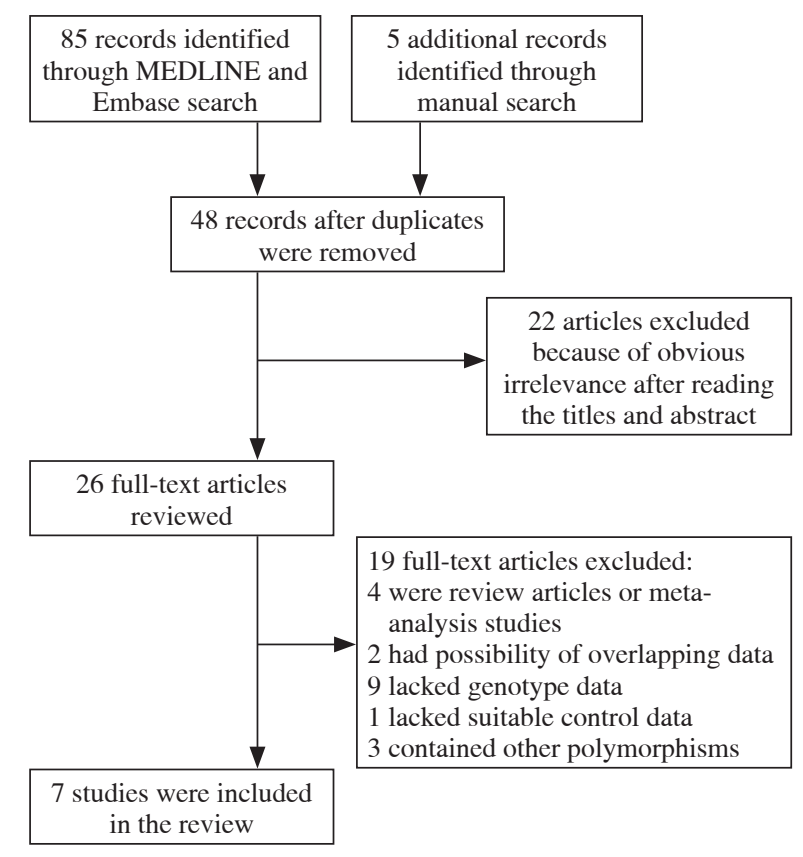

Fig. 1. Flow chart of the study's inclusion/exclusion process 


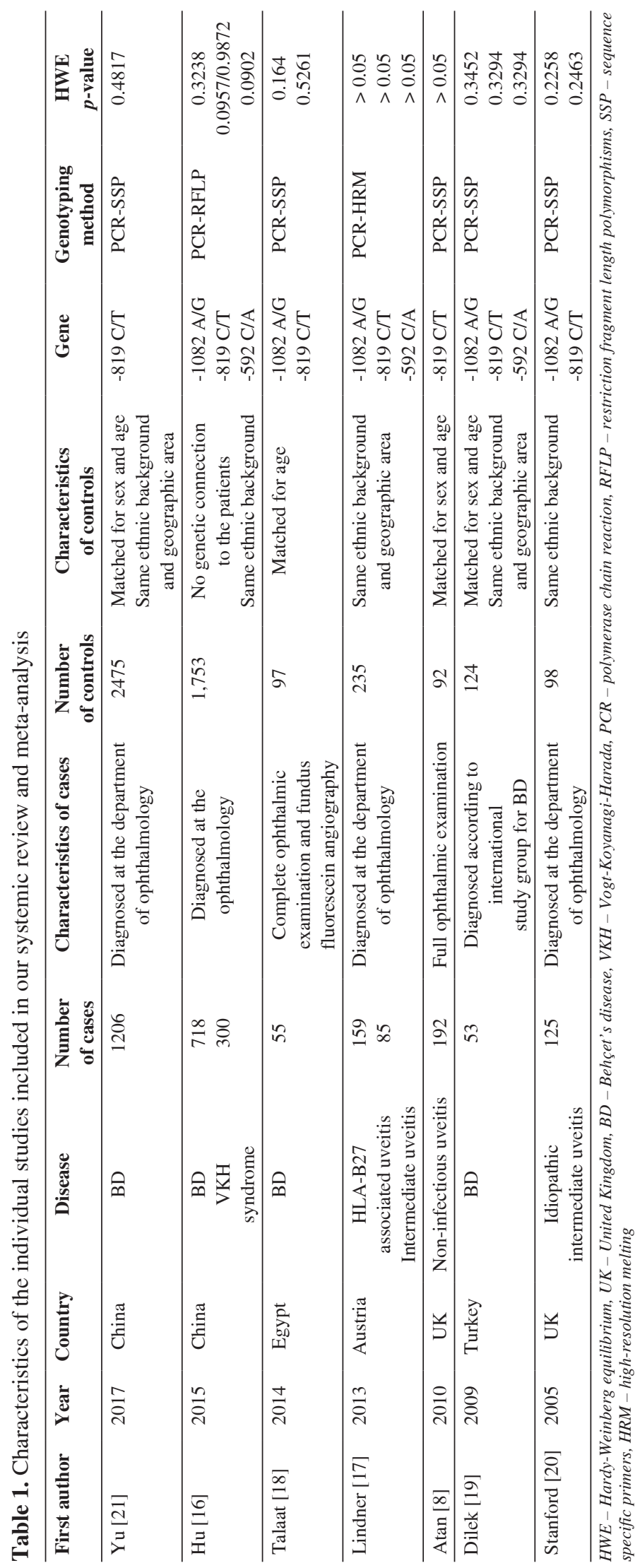

contrast and recessive and homozygous models included six studies, and the dominant model included eight studies.

The case group included all types of autoimmune uveitis, and uveitis was diagnosed in the department of ophthalmology or using objective tools. The control group was healthy, mostly of the same ethnicity or geographic area, and matched for age and sex, or had similar characteristics to the case groups. The selected characteristics of these studies with respect to the associations between IL-10 polymorphisms and uveitis are summarised in Table 1.

\section{Meta-analysis of the IL-10 -1082 A/G and $\mathbf{- 8 9 2}$ $\mathrm{C} / \mathrm{T}$ polymorphisms and susceptibility of autoimmune uveitis}

The meta-analysis revealed no association between uveitis and the IL-10 -1082 A allele (OR $=0.91,95 \%$ $\mathrm{CI}=0.64-1.30, p=0.62)$. In addition, no association was found using the pooled results for all of the models of the IL-10 -1082 A/G polymorphisms. The meta-analysis revealed significant association between uveitis and the $-892 \mathrm{C}$ allele (OR $=0.81,95 \% \mathrm{CI}=0.67-0.98, p=0.03$; Fig. 2A). In addition, significant association was found in homozygous models $(\mathrm{OR}=0.58,95 \% \mathrm{CI}=0.36-0.92, p=0.02$; Fig. 2D). However, the dominant and recessive models of the IL-10 -819 C/T polymorphisms showed no association between uveitis and each genotype (Figs. $2 \mathrm{~B}$ and $2 \mathrm{C}$ ). The meta-analysis findings concerning the associations between IL-10 polymorphisms and uveitis are provided in Table 2.

Four studies were conducted on BD, and subgroup analysis was conducted on BD. In the IL-10 -1082 A/G polymorphisms there were no associations among uveitis of $\mathrm{BD}$, allelic contrast, and any of the models. However, there were significant associations among uveitis of $\mathrm{BD}$, allelic contrast, and all of the models of the IL-10 -819 C/T polymorphisms (Table 3 and Fig. 3).

\section{Heterogeneity and publication bias}

Some heterogeneity was found in this meta-analysis of the IL-10 -1082 A/G and -819 C/T polymorphisms (Table 2). Except for the dominant model of $-1082 \mathrm{~A} / \mathrm{G}$, there was no change in the effect model used. Publication bias was examined using a funnel plot. Figure 4 shows the funnel plots for positive results. The funnel plots did not appear to be exactly symmetric.

\section{Discussion}

IL-10 is a potent anti-inflammatory cytokine produced by $\mathrm{T}$ cells, macrophages, and retinal cells [22]. Functional studies of IL-10 promoter polymorphisms have suggested that some genotypes or haplotypes are linked to down-regulation of IL-10 production [23]. This meta-analysis indicated that IL-10 -819 C/T polymorphisms were significantly associated with susceptibility to autoimmune uveitis. 


\begin{tabular}{|c|c|c|c|c|c|c|}
\hline $\mathbf{A}$ & Uve & & & & & Odds ratio \\
\hline Study or subgroup & Events & Total & Events & Total & Weight & M-H, random, 95\% CI \\
\hline Stanford et al. & 202 & 250 & 148 & 196 & $10.8 \%$ & $1.36(0.87,2.15)$ \\
\hline Dan et al. & 76 & 106 & 175 & 248 & $9.4 \%$ & $1.06(0.64,1.75)$ \\
\hline Talat et al. & 77 & 110 & 141 & 194 & $9.1 \%$ & $0.88(0.52,1.47)$ \\
\hline Hu et al. (VKH) & 194 & 600 & 248 & 700 & $20.0 \%$ & $0.87(0.69,1.10)$ \\
\hline Hu et al. (BD) & 367 & 1436 & 1259 & 3506 & $24.8 \%$ & $0.61(0.53,0.70)$ \\
\hline Yu et al. & 631 & 2412 & 1630 & 4950 & $26.1 \%$ & $0.72(0.65,0.80)$ \\
\hline Total $(95 \%$ CI) & & 4914 & & 9794 & $100.0 \%$ & $0.81(0.67,0.98)$ \\
\hline Total events & 1547 & & & & & \\
\hline Heterogeneity: $\mathrm{Tau}^{2}$ & $0.03, \chi^{2}$ & 8.38, & $f=5(t$ & 03) & $73 \%$ & \\
\hline Test for overall effec & $z=2$. & $=0$. & & & & \\
\hline
\end{tabular}

B

Uveitis

Control

Odds ratio

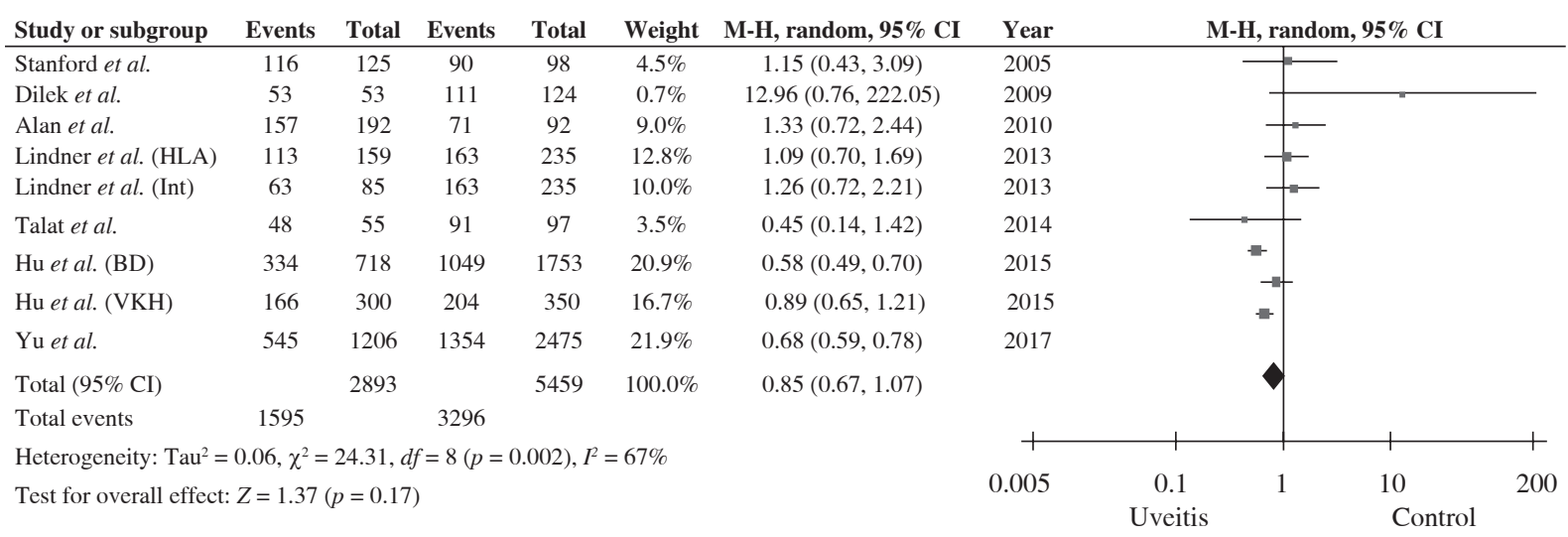

C Uveitis Control Odds ratio

Study or subgroup Events Total Events Total Weight M-H, random, 95\% CI Year

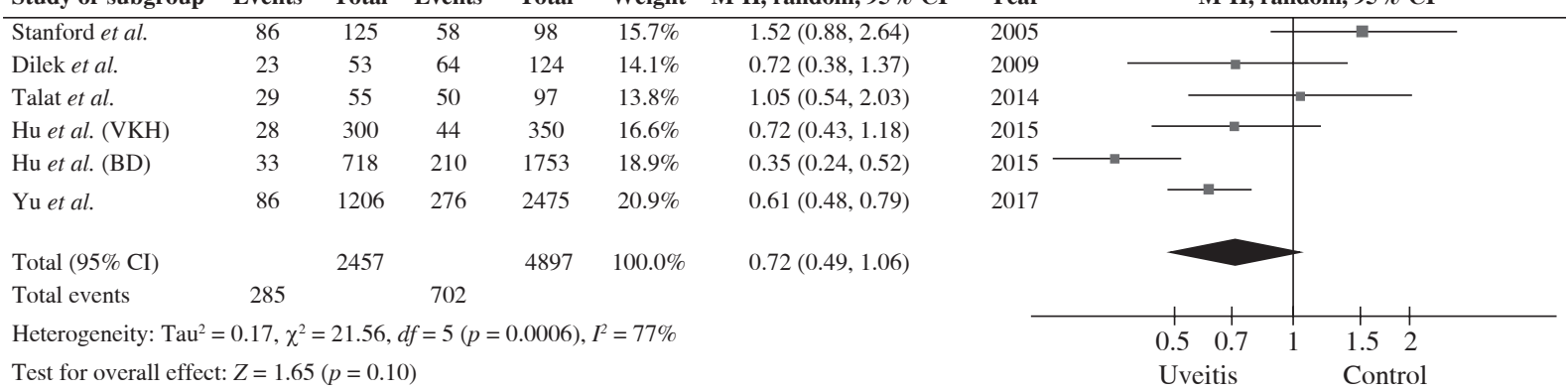

Test for overall effect: $Z=1.65(p=0.10)$

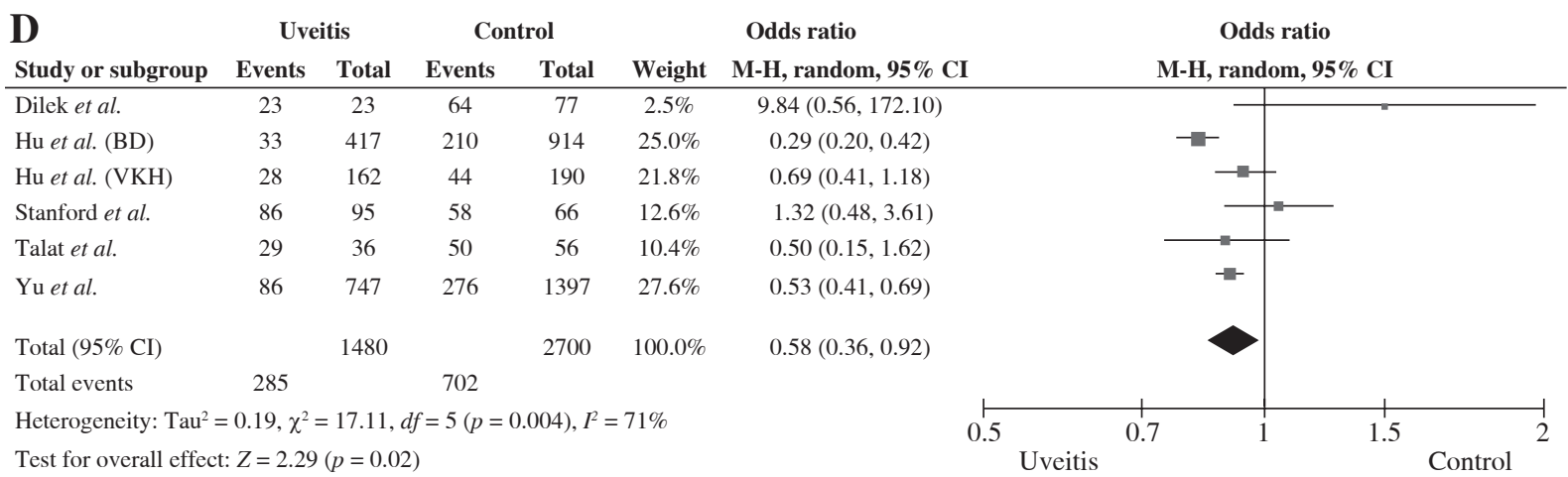

Fig. 2. ORs and 95\% CIs of the IL-10 -819 C/T polymorphisms and autoimmune uveitis. Allelic contrast (A), dominant (B), recessive (C), and homozygous (D) models 
Table 2. Meta-analysis of the associations between the IL-10 polymorphisms and autoimmune uveitis

\begin{tabular}{|c|c|c|c|c|c|c|c|}
\hline \multirow[t]{2}{*}{ Polymorphism } & & \multicolumn{3}{|c|}{ Test of association } & \multicolumn{3}{|c|}{ Test of heterogeneity } \\
\hline & & OR & $95 \% \mathrm{CI}$ & $p$-value & Model & $p$-value & $I^{2}(\%)$ \\
\hline \multirow[t]{4}{*}{$-1082 \mathrm{~A} / \mathrm{G}$} & A vs. $G$ & 0.91 & $0.64-1.30$ & 0.62 & $\mathrm{R}$ & 0.01 & 69 \\
\hline & $\mathrm{AA}+\mathrm{AG}$ vs. GG & 0.86 & $0.66-1.10$ & 0.23 & $\mathrm{~F}$ & 0.22 & 27 \\
\hline & AA vs. $A G+G G$ & 0.78 & $0.35-1.73$ & 0.54 & $\mathrm{R}$ & $<0.0001$ & 87 \\
\hline & AA vs. GG & 0.66 & 0.39-1.09 & 0.10 & $\mathrm{~F}$ & 0.49 & 0 \\
\hline \multirow[t]{4}{*}{$-819 \mathrm{C} / \mathrm{T}$} & $\mathrm{C}$ vs. T & 0.81 & $0.67-0.98$ & 0.03 & $\mathrm{R}$ & 0.003 & 73 \\
\hline & $\mathrm{CC}+\mathrm{CT}$ vs. TT & 0.85 & $0.67-1.07$ & 0.17 & $\mathrm{R}$ & 0.002 & 67 \\
\hline & $\mathrm{CC}$ vs. $\mathrm{CT}+\mathrm{TT}$ & 0.72 & $0.49-1.06$ & 0.10 & $\mathrm{R}$ & 0.0006 & 77 \\
\hline & CC vs. TT & 0.58 & $0.36-0.92$ & 0.02 & $\mathrm{R}$ & 0.004 & 71 \\
\hline
\end{tabular}

Table 3. Meta-analysis of the associations between the IL-10 polymorphisms and autoimmune uveitis of Behçet's disease

\begin{tabular}{lcccccccc}
\hline Polymorphism & \multicolumn{3}{c}{ Test of association } & \multicolumn{3}{c}{ Test of heterogeneity } \\
\hline$-1082 \mathrm{~A} / \mathrm{G}$ & $\mathbf{O R}$ & $\mathbf{9 5 \%}$ CI & $\boldsymbol{p}$-value & Model & $\boldsymbol{p}$-value & $\boldsymbol{I}^{\mathbf{2}(\boldsymbol{\%})}$ \\
\hline & A vs. G & 0.90 & $0.46-1.77$ & 0.76 & $\mathrm{R}$ & 0.002 & 84 \\
\hline & AA+AG vs. GG & 0.91 & $0.18-4.55$ & 0.91 & $\mathrm{R}$ & 0.005 & 66 \\
\hline & AA vs. AG+GG & 0.69 & $0.19-2.47$ & 0.57 & $\mathrm{R}$ & $<0.00001$ & 92 \\
\hline$-819 \mathrm{C} / \mathrm{T}$ & AA vs. GG & 0.53 & $0.25-1.13$ & 0.10 & $\mathrm{~F}$ & 0.32 & 12 \\
\hline & C vs. T & 0.71 & $0.60-0.84$ & $<0.0001$ & $\mathrm{R}$ & 0.07 & 58 \\
\hline & CC+CT vs. TT & 0.64 & $0.51-0.80$ & 0.0001 & $\mathrm{R}$ & 0.09 & 54 \\
\hline & CC vs. CT+TT & 0.60 & $0.40-0.90$ & 0.01 & $\mathrm{R}$ & 0.02 & 71 \\
\hline
\end{tabular}

Autoimmune uveitis comprises a heterogeneous group of disorders diagnosed using clinical characteristics and is accompanied by multiple systemic diseases; therefore, multiple genetic factors and cytokines may affect the development of uveitis in each condition. This meta-analysis contained various types of uveitis including BD, VKH syndrome, HLA-B27-associated uveitis, intermediate uveitis, sarcoidosis, sympathetic ophthalmia, and white dot with or without inflammation.

In this study, the $-819 \mathrm{C}$ allele and $\mathrm{CC}$ genotype were associated with low risk of uveitis. Although not statistically significant, the $\mathrm{C}$ allele in the dominant and recessive models also showed a trend toward lower risk of uveitis. In uveitis of $\mathrm{BD}$, the $-819 \mathrm{C} / \mathrm{T}$ allelic contrast and all genotype models showed that the $\mathrm{C}$ allele was significantly associated with lower risk. This result is consistent with the results from the previous meta-analysis on BD [10]. However, for diseases other than BD, subgroup analysis was not performed because the number of studies was less than two. Therefore, further studies on these diseases are necessary.

Several previous studies have suggested that IL-10 polymorphisms are related to the clinical manifestations of uveitis, such as severity, recurrence rate, and treatment response [20, 22]. In addition, pro-inflammatory cytokines influence autoimmune uveitis, and blocking agents of the pro-inflammatory cytokine pathway, especially TNF- $\alpha$ inhibitors, have been used to treat this disorder [2, 5, 24, 25]. TNF- $\alpha$ blocking agents are reportedly effective in treating AS, BD, and sarcoidosis accompanying uveitis [26-28]. As a result, IL-10 may down-regulate TNF- $\alpha$ production and has been correlated with disease severity [25-29]; thus, IL-10 can affect disease severity and the treatment response of uveitis.

As with any meta-analysis, there were some limitations to this study, and its results should be interpreted with caution. First, each case of uveitis has different clinical and pathological characteristics. The heterogeneity and confounding factors may have distorted the meta-analysis. We did not perform a subgroup analysis by disease category except BD due to the small number of published studies available on this topic. Second, stratification for ethnicity was also impossible because of the small number of cases. Therefore, well-designed and large-scale studies are required for further analyses. Third, IL-10 polymorphisms may be associated with disease severity and treatment response as well as susceptibility; however, we did not perform a meta-analysis to determine this association. Fourth, we did not conduct a haplotype study because only 


\begin{tabular}{|c|c|c|c|c|c|c|c|c|c|}
\hline \multirow{3}{*}{$\begin{array}{l}\text { A } \\
\text { Study or subgroup } \\
\text { Dilek } \text { et } a l\end{array}$} & \multicolumn{2}{|c|}{ Uveitis } & \multicolumn{2}{|c|}{ Control } & \multicolumn{3}{|c|}{ Odds ratio } & \multirow{2}{*}{\multicolumn{2}{|c|}{$\begin{array}{c}\text { Odds ratio } \\
\text { M-H, random, } 95 \% \text { CI }\end{array}$}} \\
\hline & Events & Total & Events & Total & Weight & M-H, random, $95 \%$ CI & Year & & \\
\hline & 76 & 106 & 175 & 248 & $9.2 \%$ & $1.06(0.64,1.75)$ & 2009 & & \\
\hline Talat et al. & 77 & 110 & 141 & 194 & $8.8 \%$ & $0.88(0.52,1.47)$ & 2014 & & \\
\hline Hu et al. (BD) & 367 & 1436 & 1259 & 3506 & $38.9 \%$ & $0.61(0.53,0.70)$ & 2015 & 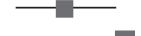 & \\
\hline Yu et al. & 631 & 2412 & 1630 & 4950 & $43.1 \%$ & $0.72(0.65,0.80)$ & 2017 & & \\
\hline Total (95\% CI) & & 4064 & & 8898 & $100.0 \%$ & $0.71(0.60,0.84)$ & & & \\
\hline Total events & 1151 & & 3205 & & & & & & \\
\hline Heterogeneity: $\mathrm{Tau}^{2}$ & $=0.01, \chi^{2}$ & $=7.12$, & $f=3(\mathrm{p}=$ & $0.07), I$ & $=58 \%$ & & 0.5 & 0.7 & 1.5 \\
\hline
\end{tabular}

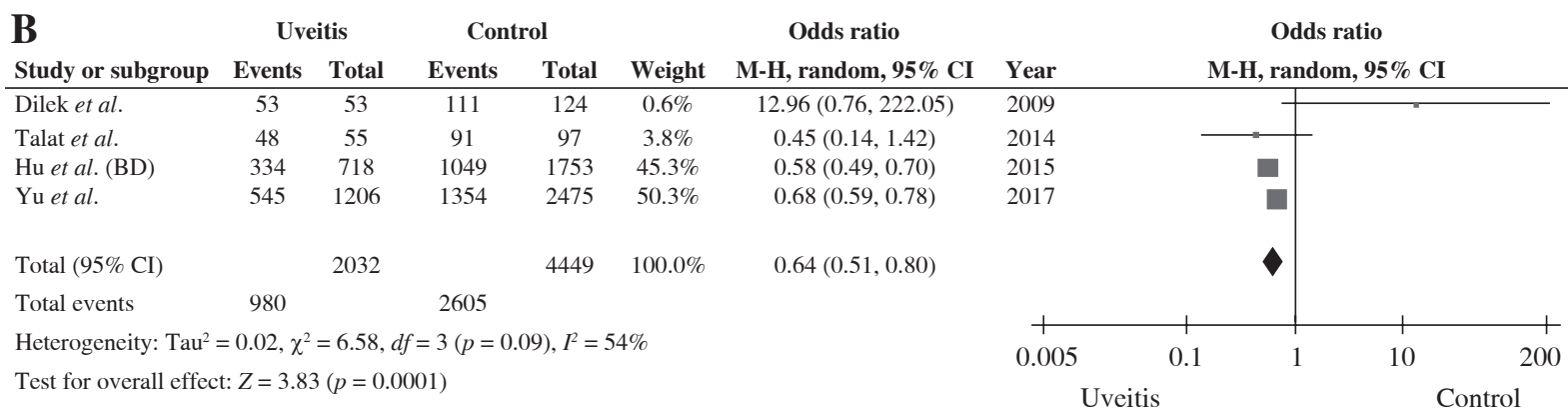

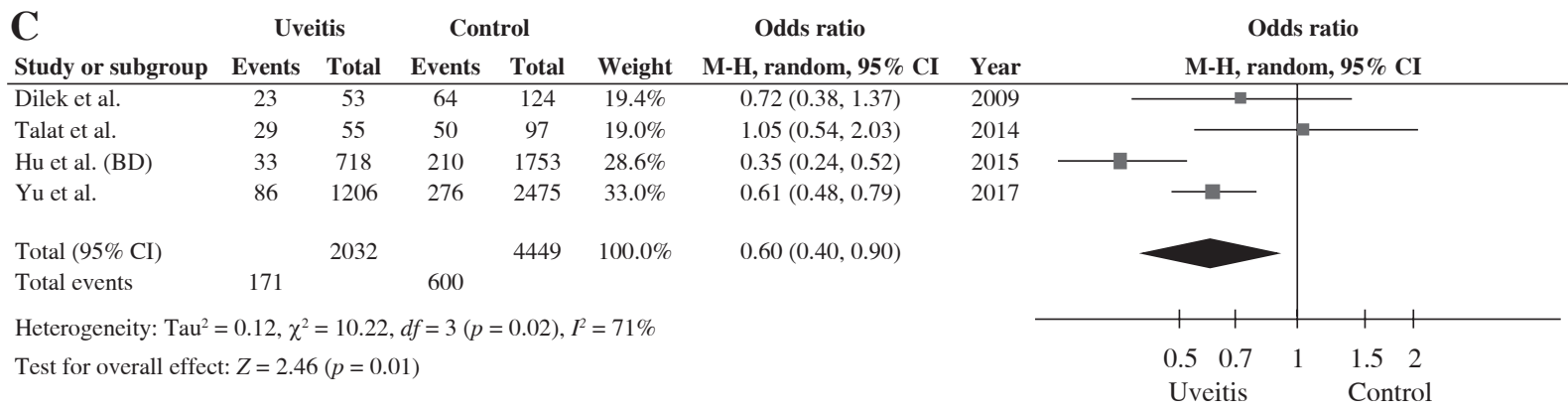

\begin{tabular}{|c|c|c|c|c|c|c|c|c|c|}
\hline \multirow{2}{*}{$\begin{array}{l}\text { D } \\
\text { Study or subgroup }\end{array}$} & \multicolumn{2}{|c|}{ Uveitis } & \multicolumn{2}{|c|}{ Control } & \multicolumn{3}{|c|}{ Odds ratio } & \multirow{2}{*}{\multicolumn{2}{|c|}{$\begin{array}{c}\text { Odds ratio } \\
\text { M-H, random, } 95 \% \mathrm{Cl}\end{array}$}} \\
\hline & Events & Total & Events & Total & Weight & M-H, random, $95 \%$ CI & Year & & \\
\hline & 23 & 23 & 64 & 77 & $3.9 \%$ & $9.84(0.56,172.10)$ & 2009 & & $=-$ \\
\hline Hu et al. (BD) & 33 & 417 & 210 & 914 & $38.1 \%$ & $0.29(0.20,0.42)$ & 2014 & - & \\
\hline Talat et al. & 29 & 36 & 50 & 56 & $16.2 \%$ & $0.50(0.15,1.62)$ & 2015 & & - \\
\hline Yu et al. & 86 & 747 & 276 & 1397 & $41.8 \%$ & $0.53(0.41,0.69)$ & 2017 & 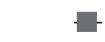 & \\
\hline Total $(95 \% \mathrm{CI})$ & & 1223 & & 2444 & $100.0 \%$ & $0.47(0.26,0.84)$ & & & \\
\hline Total events & 171 & & 600 & & & & & & \\
\hline $\begin{array}{l}\text { Heterogeneity: } \mathrm{Tau}^{2}= \\
\text { Test for overall effec }\end{array}$ & $\begin{array}{l}=0.20, \chi^{2} \\
\mathrm{t}: Z=2.53\end{array}$ & $\begin{array}{l}=11.04 \\
(p=0 .\end{array}$ & $d f=3(p$ & $=0.01)$ & $=73 \%$ & & & $\begin{array}{cc}0.5 & 0.7 \\
\text { Uveitis }\end{array}$ & $\begin{array}{lll}1 & 1.5 & 2 \\
& \text { Control }\end{array}$ \\
\hline
\end{tabular}

Fig. 3. ORs and $95 \%$ CIs of the IL-10 -819 C/T polymorphisms and autoimmune uveitis of BD. Allelic contrast (A), dominant $(\mathbf{B})$, recessive $(\mathbf{C})$, and homozygous (D) models

one study contained haplotype data. Finally, publication bias could not be completely excluded.

The pathogenesis of autoimmune uveitis remains incompletely understood; however, cytokines seem to be critical mediators in this process. Cytokines act in a complex manner to mediate immune responses, and IL-10 has an anti-inflammatory effect on the inflammatory process [30].

This meta-analysis showed that the -1082 A/G polymorphisms of IL-10 were not associated with autoimmune uveitis, but the $-819 \mathrm{C} / \mathrm{T}$ polymorphisms were significantly associated with uveitis. However, these results should be interpreted with caution given the study's limitations. Further studies with larger sample sizes that also include polymorphisms of other cytokines are needed.

The authors declare no conflict of interest. 

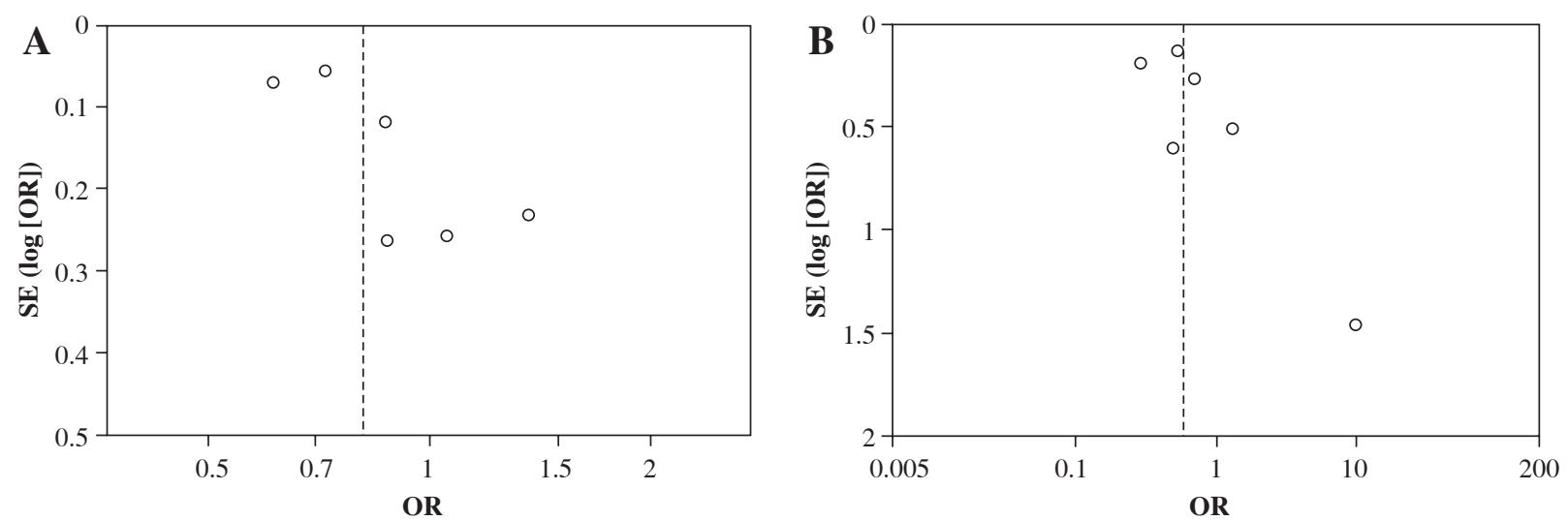

Fig. 4. Funnel plot of autoimmune uveitis and the $-819 \mathrm{C}$ allele (A) and CC vs. TT polymorphisms (B)

\section{References}

1. Selmi C (2014): Diagnosis and classification of autoimmune uveitis. Autoimmun Rev 13: 591-594.

2. Agrawal R, Iyer J, Connolly J, et al. (2014): Cytokines and biologics in non-infectious autoimmune uveitis: bench to bedside. Indian J Ophthalmol 62: 74-81.

3. Horai R, Caspi RR (2011): Cytokines in autoimmune uveitis. J Interferon Cytokine Res 31: 733-744.

4. Lin P (2015): Targeting interleukin-6 for noninfectious uveitis. Clin Ophthalmol 9: 1697-1702.

5. Cordero-Coma M, Sobrin L (2015): Anti-tumor necrosis factor-alpha therapy in uveitis. Surv Ophthalmol 60: 575-589.

6. Takeuchi M (2013): A systematic review of biologics for the treatment of noninfectious uveitis. Immunotherapy 5: 91-102.

7. Ghasemi H, Ghazanfari T, Yaraee R, et al. (2012): Roles of IL-10 in ocular inflammations: a review. Ocul Immunol Inflamm 20: 406-418.

8. Atan D, Fraser-Bell S, Plskova J, et al. (2010): Cytokine polymorphism in noninfectious uveitis. Invest Ophthalmol Vis Sci 51: 4133-4142.

9. Lv C, Wang Y, Wang J, et al. (2011): Association of Interleukin-10 gene polymorphisms with ankylosing spondylitis. Clin Invest Med 34: E370.

10. Jung JH, Song GG, Lee YH (2015): Meta-Analysis of Associations Between Interleukin-10 Polymorphisms and Susceptibility to Vasculitis. Immunol Invest 44: 553-565.

11. Jabs DA, Nussenblatt RB, Rosenbaum JT (2005): Standardization of uveitis nomenclature for reporting clinical data. Results of the First International Workshop. Am J Ophthalmol 140: 509-516.

12. Moher D, Liberati A, Tetzlaff J, et al. (2010): Preferred reporting items for systematic reviews and meta-analyses: the PRISMA statement. Int J Surg 8: 336-341.

13. Higgins JP, Thompson SG (2002): Quantifying heterogeneity in a meta-analysis. Stat Med 21: 1539-1558.

14. Egger M, Smith GD, Phillips AN (1997): Meta-analysis: principles and procedures. BMJ 315: 1533-1537.

15. DerSimonian R, Laird N (1986): Meta-analysis in clinical trials. Control Clin Trials 7: 177-188.

16. Hu J, Hou S, Zhu X, et al. (2015): Interleukin-10 gene polymorphisms are associated with Behcet's disease but not with Vogt-Koyanagi-Harada syndrome in the Chinese Han population. Mol Vis 21: 589-603.

17. Lindner E, Steinwender G, Plainer S, et al. (2013): Role of IL-10 gene polymorphisms in intermediate and HLA-B27-associated uveitis. Acta Ophthalmol 91: e415-417.
18. Talaat RM, Ashour ME, Bassyouni IH, et al. (2014): Polymorphisms of interleukin 6 and interleukin 10 in Egyptian people with Behcet's disease. Immunobiology 219: 573-582.

19. Dilek K, Ozcimen AA, Saricaoglu H, et al. (2009): Cytokine gene polymorphisms in Behcet's disease and their association with clinical and laboratory findings. Clin Exp Rheumatol 27: S73-78.

20. Stanford MR, Vaughan RW, Kondeatis E, et al. (2005): Are cytokine gene polymorphisms associated with outcome in patients with idiopathic intermediate uveitis in the United Kingdom? Br J Ophthalmol 89: 1013-1016.

21. Yu H, Zheng M, Zhang L, et al. (2017): Identification of susceptibility SNPs in IL10 and IL23R-IL12RB2 for Behcet's disease in Han Chinese. J Allergy Clin Immunol 139: 621-627.

22. Atan D, Turner SJ, Kilmartin DJ, et al. (2005): Cytokine gene polymorphism in sympathetic ophthalmia. Invest Ophthalmol Vis Sci 46: 4245-4250.

23. Crawley E, Kay R, Sillibourne J, et al. (1999): Polymorphic haplotypes of the interleukin-10 5' flanking region determine variable interleukin-10 transcription and are associated with particular phenotypes of juvenile rheumatoid arthritis. Arthritis Rheum 42: 1101-1108.

24. Kim M, Won JY, Choi SY, et al. (2016): Anti-TNFalpha Treatment for HLA-B27-Positive Ankylosing Spondylitis-Related Uveitis. Am J Ophthalmol 170: 32-40.

25. Levy-Clarke G, Jabs DA, Read RW, et al. (2014): Expert panel recommendations for the use of anti-tumor necrosis factor biologic agents in patients with ocular inflammatory disorders. Ophthalmology 121: 785-796.

26. Liu W, Wu YH, Zhang L, et al. (2016): Efficacy and safety of TNF-alpha inhibitors for active ankylosing spondylitis patients: Multiple treatment comparisons in a network meta-analysis. Sci Rep 6: 32768.

27. Desbois AC, Addimanda O, Bertrand A, et al. (2016): Efficacy of Anti-TNFo in Severe and Refractory Neuro-Behcet Disease: An Observational Study. Medicine (Baltimore) 95: e3550.

28. Amber KT, Bloom R, Mrowietz U, et al. (2015): TNF-alpha: a treatment target or cause of sarcoidosis? J Eur Acad Dermatol Venereol 29: 2104-2111.

29. Amir O, Rogowski O, David M, et al. (2010): Circulating interleukin-10: association with higher mortality in systolic heart failure patients with elevated tumor necrosis factor-alpha. Isr Med Assoc J 12: 158-162.

30. Wallace GR, Kondeatis E, Vaughan RW, et al. (2007): IL-10 genotype analysis in patients with Behcet's disease. Hum Immunol 68: 122-127. 